\title{
Risk Prediction and Prognosis of Invasive Fungal Disease in Hematological Malignancies Patients Complicated with Bloodstream Infections
}

This article was published in the following Dove Press journal: Cancer Management and Research

\author{
Han Xiao' \\ Yishu Tang ${ }^{2}$ \\ Qian Cheng' \\ Jing Liu' \\ Xin $\mathrm{Li}^{1}$ \\ 'Department of Hematology, The Third \\ Xiangya Hospital, Central South \\ University, Changsha, Hunan, People's \\ Republic of China; ${ }^{2}$ Department of \\ Emergency, The Third Xiangya Hospital, \\ Central South University, Changsha, \\ Hunan, People's Republic of China
}

\begin{abstract}
Purpose: In patients with hematologic malignancies (HM), bloodstream infections (BSI) and invasive fungal disease (IFD) remain important complications causing considerable mortality and morbidity. At present, the morbidity of IFD and the strategies to initiate antifungal treatment in HM patients with BSI remain unclear.
\end{abstract}

Patients and Methods: Patient characteristics, infection-related variables, and therapyrelated features of $1374 \mathrm{HM}$ patients with proven BSI from three hospitals were reviewed to investigate the epidemiology, risk factors and prognosis of IFD.

Results: The morbidity of proven and probable IFD in HM patients with BSI was $11.2 \%$, and the mortality of those patients was $40.5 \%$. Existing IFD risk scores were not accurate enough in distinguishing these patients benefiting from antifungal prophylaxis. Multivariate logistic regression identified age $>45$ years, profound neutropenia, hypoproteinemia, and use of vasopressors as independent variables associated with IFD morbidity in HM patients with BSI. In patients with proven and probable IFD patients, age $>45$ years, Pitt bacteremia score $>3$, use of vasopressors, abnormal blood coagulation, and initiation of antifungal therapy within $72 \mathrm{hrs}$ after the onset of fever were independent prognostic factors. The mortality was significantly reduced in patients with high-risk factors of IFD if they initiate antifungal treatment within $72 \mathrm{hrs}$ after the onset of fever compared to the patients not.

Conclusion: The morbidity and mortality of IFD increase significantly in HM patients with BSI. Early antifungal therapy may improve prognosis in HM patients with BSI complicated with IFD risk factors.

Keywords: invasive fungal diseases, hematologic malignancies, bloodstream infections, risk scores, prognosis

\section{Introduction}

Unlike solid tumors, hematological malignancies patients (HM) are susceptible to prolonged neutropenia and immunosuppression during treatment, with a very high risk of infection, the incidence and mortality of bloodstream infections (BSI) are significantly higher than other tumors. ${ }^{1}$ Epidemiology suggests a predominance of bacterial etiologic agents in BSI and a low detection rate of fungi. ${ }^{2,3}$

Our previous study has suggested that the prognosis of bacterial bloodstream infection has been greatly improved in recent years due to the standardized treatment of HM patients with febrile neutropenia, and the death rate has decreased from $23.8 \%$ to $14.4 \%$. ${ }^{4}$ However, the mortality of fungal BSI still remains high. ${ }^{5,6}$ Other than fungal infections with clear etiological evidence, the mortality of clinically
Department of Hematology, The Third

Xiangya Hospital, Central South

University, Changsha, Hunan, People's

Republic of China

Tel/Fax +86-73I-886I80I4

Email lixiner1975@I63.com 
diagnosed invasive fungal disease is also very high. ${ }^{7,8}$ How to improve the survival of those patients remains a great challenge at present.

In HM patients, BSI should be highly suspected in case of febrile neutropenia. In Infectious Diseases Society of America (IDSA) guideline, all patients who present with fever and neutropenia should be treated swiftly and broadly with antibiotics to treat both gram-positive and gramnegative pathogens. ${ }^{9}$ With regard to the optimal duration of antifungal therapy in patients with neutropenic fever, current guidelines recommend the empirical antifungal treatment for patients with persistent fever should last for 4-7 days after broad-spectrum antibiotic chemotherapy. ${ }^{10}$ Based on the 2008 European Organization for the Research and Treatment of Cancer/Mycoses Study Group (EORTC/ MSG) criteria, immunocompromised patients with cancer and patients undergoing HSCT are diagnosed with "proven," "probable," and "possible" invasive fungal disease. ${ }^{11}$ In case of an early initiation of antifungal therapy, pre-emptive treatment was recommended as a diagnostic-driven therapy for patients with persistent fever together with diagnostic workup showing suspicious findings prior to the initiation of antifungal treatment. However, HM patient may not meet to the criterion of possible, probable, or proven IFD at the beginning of the fever episode, but may meet the criterion at a later time, ${ }^{12}$ thus delaying the timing of antifungal therapy and affecting the prognosis of patients. On the other hand, excessively aggressive antifungal prophylaxis results in unnecessary side effects and high medical costs, thereby increasing the incidence of drug resistance. ${ }^{13-15}$ As indicated in the NCCN Guidelines for the Treatment and Prevention of Cancer-Related Infections, antifungal prophylaxis should not be used routinely in all patients with neutropenia. ${ }^{16}$ Therefore, the best time to initiate fungal treatment in those HM patients remains unclear in case of suspected of BSI.

For the above purpose, we collected the relevant clinical data of HM patients with BSI to investigate the occurrence, risk factors and prognostic factors of IFD in those patients and may help clinicians to decide the initiation of antifungal therapy in HM patients with suspected BSI.

\section{Materials and Methods}

\section{Setting and Study Design}

In this retrospective study, we collected relevant clinical data of the patients from the hematology wards of three affiliated teaching hospitals in Hunan Province from January 2010 to July 2018. The subjects of the present study were enrolled among the hematological patients with positive blood cultures recorded by the computer system in the microbiology laboratory of the three hospitals. For patients with multiple positive cultures with the same morphology and sensitivity, only they were only counted once. Exclusion criteria: (1) patients diagnosed with nonhematological malignancies; (2) blood culture contamination; (3) failure to attend follow-up; and (4) no assessment of fungal infection during treatment. The obtained data on patients with positive blood cultures included baseline characteristics, hematological diagnosis, chemotherapy, clinical features, antifungal therapy, and epidemiological and treatment-related potential risk factors for IFD. The study was conducted in accordance with the Declaration of Helsinki, International Conference on Harmonization/ Good Clinical Practice and nationally mandated ethical requirements. The study protocol and informed consent document were reviewed and approved by the Ethics Committee of the Central South University.

\section{Definitions}

The data collected included subject's characteristics at baseline, hematologic diagnosis and chemotherapy, risk factors for IFD, clinical features of IFD, fungal test results, antifungal prophylaxis and treatment, and survival status at discharge. IFD was classified as proven, probable or possible in accordance with the 2008 version of EORTC/MSG criteria. The management of the patients receiving antifungal prophylaxis and/or therapy was recorded, including the date and nature of the change in treatment and survival status at discharge. Each inpatient hospitalization represented one case, and if a patient was re-hospitalized and received another round of treatment, he/she was also considered a new and separate case.

\section{Statistical Analysis}

A statistical analysis was performed using the SPSS 22.0 software package. The cut-off values of continuous variables were set according to clinical practice or laboratory references. A Chi-square test was used for bivariate analysis, a Logistic regression was utilized for multivariate analysis, and correlations between parameters were tested using Pearson's coefficient. $\mathrm{R}>0.6$ indicated a strong correlation and $\mathrm{R}<0.5$ indicated a weak correlation. Factors that were statistically significant in the univariate analysis were included in the multivariate analysis, and the logistic regression results were represented as ORs and their 95\% CIs. All p-values were twotailed, and $p$-values $\leq 0.05$ were considered significant. 


\section{Results}

\section{Patient Characteristics}

A total of 1033 were included (There were 1374 patients with positive blood cultures during the study; 75 with non-hematological malignancies were excluded, 60 were lost to follow-up, and 21 were not assessed for fungi during treatment.). Table 1 summarizes the demographic and clinical characteristics of the patients included in the present study. More than half of the patients were males $(56.1 \%)$ and $35.0 \%$ were over 45 years of age. Half of the patients $(50.5 \%)$ had the primary disease of acute myeloid leukemia, the majority $(68.7 \%)$ had relapsed or uncontrolled disease, a small proportion of patients $(6.0 \%$ and $10 \%$ ) concomitantly had diabetes and renal insufficiency, and $7.6 \%$ had a previous history of fungal infection. Nearly half $(45.8 \%)$ of the patients received PICC. The majority $(90.2 \%)$ were at the neutropenic phase at the time of blood culture. In terms of treatment, $89.1 \%$ of the patients received chemotherapy, $2.7 \%$ had previous history of undergoing hematopoietic stem cell transplantation, and $10.3 \%$ had the exposure to hormones/immunosuppressants. During the BSI, $20.7 \%$ of the patients required vasoactive agents to

Table I Demographic and Clinical Characteristics of Study Patients $(n=1033)$

\begin{tabular}{|l|l|}
\hline Variables & N (\%) \\
\hline Male & $580(56.1 \%)$ \\
Age $>45$ years & $362(35.0 \%)$ \\
\hline Underlying Malignancies & \\
$\quad$ Acute myeloid leukemia & $522(50.5 \%)$ \\
$\quad$ Acute lymphoblastic leukemia & $351(33.9 \%)$ \\
\hline Disease Status & \\
$\quad$ Newly-diagnosed or relapsed or uncontrolled & $710(68.7 \%)$ \\
\hline Diabetes & $63(6.0 \%)$ \\
Renal inadequacy & $104(10 \%)$ \\
Hematopoietic stem cell transplantation status & $28(2.7 \%)$ \\
Neutropenia & $932(90.2 \%)$ \\
Chemotherapy & $921(89.1 \%)$ \\
Glucocorticoid or immunosuppressive treatments & $107(10.3 \%)$ \\
Previous IFD & $79(7.6 \%)$ \\
Central venous catheter & $474(45.8 \%)$ \\
Abnormal radiographic examination & $717(69.4 \%)$ \\
Use of vasopressors & $214(20.7 \%)$ \\
IFD (proven + probable) & $116(11.2 \%)$ \\
Mortality & $216(20.9 \%)$ \\
\hline
\end{tabular}

maintain vital signs. The mortality of all patients with BSI was $20.9 \%$, and the mortality of the patients with a proven/probable IFD was $40.5 \%$.

There were 181 patients with documented IFDs during the study, including 94 patients with prove diagnosis, 22 patients with probable diagnosis, and 65 patients with possible diagnosis. The morbidity of proven/probable IFD is $11.2 \%$. The main pathogens in proven cases were Candida tropicalis ( $\mathrm{n}=53)$, Candida albicans $(\mathrm{n}=14)$, Candida parapsilosis $(\mathrm{n}=9)$, Candida glabrata $(\mathrm{n}=4)$, Candida $(\mathrm{n}=8)$, Candida famata $(\mathrm{n}=1)$, Candida krusei $(\mathrm{n}=2)$, Candida haemulonii $(\mathrm{n}=1)$, Fusarium $(\mathrm{n}=1)$, and Cryptococcus neoformans $(\mathrm{n}=1)$. Table 2 shows the susceptibility profile of all positive blood cultures; Candida tropicalis has the highest resistance rate among all Candida species, and the rate of resistance to itraconazole and fluconazole was nearly $50 \% \quad(50.9 \%$ and $45.3 \%$, respectively). Susceptibility results of all Candida strains showed that no amphotericin B-resistant strain was found.

\section{Validation of "CAESAR" Score}

Data from the present study were used to validate the "CAESAR" scores of the existing IFD prediction model for hematological malignancies. ${ }^{17}$ All patients satisfied the 7 variables in the scoring system: gender, hypoproteinemia, induction chemotherapy, severe neutropenia, prolonged neutropenia, central venous catheterization, and history of IFD. As shown in Table 3, the incidence of IFD in patients with a risk score of 0 to 10 among the HM patients with BSI was $8.2 \%$, much higher than the rate reported in the literature $(0.7 \%)$ and above the benefit margin $(3 \%$ to $5 \%)$ of prophylactic antifungal therapy. Among patients with a risk score of 11 to 15 , the IFD rate was $10.8 \%$, which was higher than the rate reported in the literature $(6.4 \%)$, and above the margin of significant benefit $(10 \%)$ of prophylactic antifungal therapy. The incidence of IFD in patients with a risk score $>15$ was $18.7 \%$, close to $17.5 \%$ reported in the literature. The use of "CAESAR" score could distinguish high-risk patients from intermediate- and low-risk patients $(\mathrm{p}<0.001$ vs 0 to 10 points, $p=0.008$ vs 11 to 15 ), but could not distinguish intermediate-risk patients and low-risk patients ( $\mathrm{P}=0.194$ vs 0 to 10 points). Therefore, IFD prophylaxis for HM patients with BSI cannot be applied to the antifungal strategy recommended in the literature that prophylaxis was given only to intermediate- and high-risk patients, and not to low-risk patients. ${ }^{17}$ 
Table 2 Resistance Rate of Candida to Antifungal Drugs

\begin{tabular}{|c|c|c|c|c|c|}
\hline $\begin{array}{l}\text { Antifungal } \\
\text { Drugs }\end{array}$ & $\begin{array}{l}\text { Candida tropicalis } \\
(n=53)\end{array}$ & $\begin{array}{l}\text { Candida albicans } \\
(n=14)\end{array}$ & $\begin{array}{l}\text { Candida parapsilosis } \\
(n=9)\end{array}$ & $\begin{array}{l}\text { Candida glabrata } \\
(n=4)\end{array}$ & $\begin{array}{l}\text { Other Candida } \\
(n=\mid 2)\end{array}$ \\
\hline Itraconazole & 27 (50.9\%) & $2(14.3 \%)$ & I (II.I\%) & $2(50.0 \%)$ & 4 (33.3\%) \\
\hline Fluconazole & 24 (45.3\%) & 2 (14.3\%) & I (II.I\%) & I (25.0\%) & $2(16.7 \%)$ \\
\hline Voriconazole & 17 (32.1\%) & $2(14.3 \%)$ & - & I (25.0\%) & I (8.3\%) \\
\hline Amphotericin B & - & - & - & - & - \\
\hline $5-\mathrm{FC}$ & - & - & - & - & I (8.3\%) \\
\hline Caspofungin & - & - & - & - & - \\
\hline Posaconazole & - & - & - & - & - \\
\hline
\end{tabular}

Note: 5-FC 5-formylcytosine.

Table 3 Distribution of "CAESAR" Score and Cumulative Incidence of IFD in HM Patients BSI

\begin{tabular}{|l|l|l|l|}
\hline $\begin{array}{l}\text { “CAESAR" } \\
\text { Score }\end{array}$ & N (\%) & $\begin{array}{l}\text { IFD Episodes (n)/ } \\
\text { Incidence (\%) }\end{array}$ & $\begin{array}{l}\text { IFD Incidence (\%) } \\
\text { in Document }\end{array}$ \\
\hline $0-10$ & $439(42.4 \%)$ & $36(8.2 \%)$ & $0.7 \%$ \\
$11-15^{*}$ & $397(38.4 \%)$ & $43(10.8 \%)$ & $6.4 \%$ \\
$>15^{* *}$ & $197(19.0 \%)$ & $37(18.7 \%)$ & $17.5 \%$ \\
\hline
\end{tabular}

Notes: ${ }^{*} \mathrm{p}=0.194$ vs $0-10 ;{ }^{* *} \mathrm{p}<0.01$ vs $0-10, \mathrm{p}=0.008$ vs $\mid \mathrm{I}-15$.

\section{Analysis of Factors Affecting IFD in HM Patients with BSI}

There has been currently no guideline on the timing of relevant antifungal therapy for HM patients with BSI, and we need therefore to investigate the antifungal strategy applicable to such patients. Efforts should be made to find the factors that may affect the development of IFD in such patients. We included the literature-reported factors with a possible correlation with fungal infection for analysis. As shown in Supplementary Table 1, there were no statistically significant difference between IFD and non-IFD groups in terms gender, type and status of underlying diseases, presence or absence of previous history of hematopoietic stem cell transplantation, chemotherapy, immunosuppressant use, central venous catheterization and duration of granulocytopenia $(\mathrm{P}>0.05)$. Compared to the non-IFD group, the IFD group had a higher proportion of patients over 45 years of age $(43.1 \%$ vs $34.0 \%$, p = 0.043 ), had a higher proportion of patients with renal insufficiency $(17.2 \%$ vs $9.2 \%, p=0.006)$, had a larger number of patients with a Charlson index score of more than 3 points and a Pitt score of more than 3 points $(21.6 \%$ vs $11.7 \%, p=0.003,35.3 \%$ vs $21.3 \%, p=0.001$, respectively), had a higher likelihood of developing severe neutropenia $(90.5 \%$ vs $81.4 \%, \mathrm{p}=0.015)$, and had more patients requiring vasoactive medication to correct shock
(35.5\% vs $18.9 \%, \mathrm{p}<0.001)$. Subsequently, the variables that were statistically significant by univariate analysis as indicated by $\mathrm{P} \leq 0.05$ were included in the multivariate regression analysis, including age $>45$ years, renal insufficiency, Charlson index core $>3$ points, Pitt bacteremia score $>3$, severe neutropenia, use of vasoactive drugs, anemia (hemoglobin $<55 \mathrm{~g} / \mathrm{L}$ ), hypoproteinemia (albumin $<30 \mathrm{~g} / \mathrm{L}$ ), abnormal liver function (ALT $\geq 60 \mu / \mathrm{L}$ ), and abnormal coagulation function (APTT $\geq 43 \mathrm{~s}$ ). A multivariate logistic regression analysis showed that, age $>45$ years (OR: $1.531 ; 95 \% \mathrm{CI}: 1.007$ to 2.327 ; $\mathrm{P}=$ 0.046), severe neutropenia (OR: $1.989 ; 95 \%$ CI: 1.028 to $3.850 ; \mathrm{P}=0.041$ ), appearance of use of vasopressors (OR: 1.697 ; $95 \% \mathrm{CI}: 1.046$ to $2.754 ; \mathrm{P}=0.032$ ), and hypoproteinemia (OR: $1.578 ; 95 \% \mathrm{CI}: 1.006$ to $2.476 ; \mathrm{P}=0.047$ ) were independent factors affecting IFD in HM patients with BSI (Table 4).

\section{Establishment and Validation of a Risk Scoring System for the IFD Development in HM Patients with BSI}

On the basis of multivariate logistic regression analysis, 1 point was assigned if one of the following factors was satisfied: age $>45$ years, severe neutropenia, presence of use of vasopressors, and hypoproteinemia, to generate a risk score on a scale of 0 to 4 points. The risk score was calculated for each patient. The distribution and the cumulative morbidity and associated mortality of the proven/probable IFD are shown in Table 5. The morbidity of IFD in hematologic malignancy patients with bloodstream infection with a score of 0 was $5.3 \%$, and the mortality rate was $6.0 \%$; the morbidity of IFD in such patients with a score 1 to 2 points increased to $11.6 \%$, above the significant benefit margin of prophylactic antifungal therapy $(10 \%)$, and the mortality rate was $20.4 \%$; the morbidity of IFD in the patients with a score 
Table 4 Analysis of Factors Affecting IFD in BSI Patients with HM

\begin{tabular}{|c|c|c|c|c|c|c|}
\hline \multirow[t]{2}{*}{ Risk Factor } & \multirow[t]{2}{*}{$N(n=1033)$} & \multirow[t]{2}{*}{ IFD $(n=\mid 16)$} & \multicolumn{2}{|l|}{ Univariate Analysis } & \multicolumn{2}{|c|}{ Multivariate Analysis } \\
\hline & & & OR $(95 \% \mathrm{Cl})$ & $P$ value & OR $(95 \% \mathrm{Cl})$ & $P$ value \\
\hline Age $>45$ years & $362(35.0 \%)$ & $50(43.1 \%)$ & $1.469(1.093-2.174)$ & 0.043 & I.53I (I.007-2.327) & 0.046 \\
\hline Renal inadequacy & $104(10.1 \%)$ & $20(17.2 \%)$ & $2.066(1.214-3.515)$ & 0.006 & $1.229(0.678-2.228)$ & 0.497 \\
\hline Charlson Comorbidity index $>3$ & $132(12.8 \%)$ & $25(21.6 \%)$ & $2.080(1.279-3.382)$ & 0.003 & $1.159(0.665-2.021)$ & 0.603 \\
\hline Pitt bacteremia score $>3$ & $236(22.8 \%)$ & $4 \mathrm{l}(35.3 \%)$ & $2.024(1.340-3.057)$ & 0.001 & I.264 (0.78I-2.046) & 0.236 \\
\hline Profound neutropenia & $851(82.3 \%)$ & $105(90.5 \%)$ & $2.188(1.150-4.162)$ & 0.015 & I.734 (0.877-3.429) & 0.041 \\
\hline Use of vasopressors & $214(20.7 \%)$ & $4 \mathrm{l}(35.5 \%)$ & $2.35 \mathrm{I}(\mathrm{I} .552-3.56 \mathrm{I})$ & 0.000 & I.70I (I.032-2.804) & 0.032 \\
\hline $\mathrm{Hb}<55 \mathrm{~g} / \mathrm{L}$ & 421 (40.7\%) & 61 (52.6\%) & $1.716(1.164-2.529)$ & 0.006 & $1.266(0.829-1.933)$ & 0.254 \\
\hline$A L B<30 g / L$ & $546(52.9 \%)$ & 81 (69.8\%) & $2.250(1.482-3.414)$ & 0.000 & $1.625(1.036-2.55 \mathrm{I})$ & 0.047 \\
\hline $\mathrm{ALT} \geq 60 \mathrm{U} / \mathrm{L}$ & 345 (33.4\%) & $53(45.7 \%)$ & 1.801 (1.218-2.662) & 0.003 & I.379 (0.909-2.093) & 0.072 \\
\hline APTT $\geq 43 \mathrm{sec}$ & 367 (35.5\%) & $53(45.7 \%)$ & $1.616(1.094-2.386)$ & 0.015 & I.I69 (0.77-I.776) & 0.396 \\
\hline
\end{tabular}

Table 5 Distribution of Risk Fraction, Cumulative Incidence and Mortality of IFD in HM Patients with BSI

\begin{tabular}{|l|l|l|l|}
\hline $\begin{array}{l}\text { Risk } \\
\text { Score }\end{array}$ & $\begin{array}{l}\text { No. of } \\
\text { Courses (n) }\end{array}$ & $\begin{array}{l}\text { IFD Episodes (n)/ } \\
\text { Incidence (\%) }\end{array}$ & $\begin{array}{l}\text { No. of Deaths(n)/ } \\
\text { Incidence (\%) }\end{array}$ \\
\hline 0 & 283 & $15(5.3 \%)$ & $17(6.0 \%)$ \\
I-2 & 656 & $76(11.6 \%)^{*}$ & $134(20.4 \%)^{\#}$ \\
$3-4$ & 94 & $25(26.6 \%)^{* *}$ & $65(69.1 \%)^{\#}$ \\
\hline
\end{tabular}

Notes: ${ }^{*}=0.03$ Ivs. 0 ; ${ }^{*} \mathrm{p}<0.001$ vs $0, \mathrm{p}<0.001$ vs I-2 ${ }^{\#} \mathrm{p}<0.001$ vs $0 ;{ }^{\#} \mathrm{p}<0.001$ vs $0, p<0.001$ vs $I-2$.

3 to 4 points increased to $26.6 \%$, and the mortality was as high as $69.1 \%$. The scoring system not only differentiated high-risk patients from intermediate- to low-risk patients $(\mathrm{P}<$ 0.01 vs 0 points, $\mathrm{p}<0.01$ vs 1 to 2 points), but also could distinguish between intermediate-risk from low-risk patients ( $p=0.031$ vs 0 point), and there were statistically significant differences in mortality among the patients with different risk scores ( $\mathrm{p}<0.001,1$ to 2 points vs 0 point; $\mathrm{p}<0.01,3$ to 4 points vs 0 point, $\mathrm{p}<0.0013$ to 4 points vs 1 to 2 points).

\section{Analysis of Prognostic Factors After the IFD Development in HM Patients with BSI}

For HM patients with BSI known to have developed IFD, the risk factors affecting their prognosis are not yet known, and we included relevant factors for the prognosis of fungal infections reported in the literature for analysis. As shown in Supplementary Table 2, there were no statistically significant differences between the death group and the non-death group in terms gender, type of underlying disease, use of immunosuppressive agents, previous fungal infection, positive imaging, positive $G$ test, hypoalbuminemia (albumin $<30 \mathrm{~g} / \mathrm{L}$ ), abnormal liver function (ALT $\geq 60 \mu / \mathrm{L}$ ), initiation or no initiation of antifungal therapy, and use of antifungal therapy within $24 \mathrm{hrs}$ after the onset of fever $(\mathrm{P}>0.05)$. Compared to the non-death group, the death group had a higher proportion of patients over 45 years of age (55.3\% vs $34.8 \%, \mathrm{p}=0.028)$, had a higher likelihood of losing control over the underlying disease $(87.2 \%$ vs $63.8 \%, \mathrm{p}=0.001$ ), had more patients concomitantly with renal insufficiency $(31.9 \%$ vs $7.2 \%, p=0.001)$, had more patients with a Charlson index score of more than 3 points and a Pitt score of more than 3 points $(34.0 \%$ vs $13.0 \%$, $\mathrm{p}=0.007,55.3 \%$ vs $21.7 \%, \mathrm{p}<0.001$ ), and a higher proportion of patients requiring the use of vasoactive drugs to correct shock $(70.2 \%$ vs $11.6 \%, p<0.001)$. In terms of treatment, the non-death group had a higher proportion of patients who initiated antifungal therapy within $72 \mathrm{hrs}$ after the onset of fever $(60.9 \%$ vs $27.7 \%, \mathrm{p}<0.001)$. Subsequently, the variables that were statistically significant by univariate analysis as indicated by $\mathrm{P} \leq 0.05$ were included in the multivariate regression analysis, including age $>45$ years, uncontrolled primary disease, concomitant renal insufficiency, Charlson index score $>3$ points, Pitt bacteremia score $>3$ points, induction/re-induction chemotherapy, central venous catheterization, use of vasoactive drugs, anemia (hemoglobin $<55 \mathrm{~g} / \mathrm{L}$ ), abnormal blood coagulation (PT $\geq 16 \mathrm{~s}$, APTT $\geq 43 \mathrm{~s}$ ) and initiation of antifungal therapy within $72 \mathrm{hrs}$ after the onset of fever. A multivariate logistic regression analysis showed age $>45$ years (OR: 4.5 ; $95 \% \mathrm{CI}$ : 1.22-16.598; $\mathrm{p}=0.024)$, Pitt bacteremia score $>3$ points (OR: 3.899; 95\% CI: $1.080-14.082 ; \mathrm{p}=0.038$ ), the use of vasoactive drugs (OR: 16.351; 95\% CI: 3.774-70.846; $\mathrm{p}<0.001$ ), abnormal coagulation function (PT $\geq 16 \mathrm{~s}$ ) (OR: 3.805; 95\% CI: $1.026-14.114 ; p=0.046)$, and the initiation of antifungal therapy within $72 \mathrm{hrs}$ after the onset of fever (OR: 0.247; 95\% CI: $0.069-0.886 ; \mathrm{p}=0.032$ ) were independent factor 
affecting the IFD prognosis of HM patients with BSI (Table 6).

\section{Effect of Antifungal Therapy Within 72 hrs After Giving Different Risk Scores in Patients with Hematologic Malignancies Complicated with Bloodstream Infection}

Previous studies have found that risk scores might predict high-risk factors for IFD in HM patients with BSI, and that timely antifungal therapy may improve prognosis in patients with proven IFD. Therefore, we further validated the effect of antifungal therapy on prognosis in patients with high-risk factors for IFD. As shown in Table 7, we revealed that, in HM patients with BSI who did not have any risk factors and had a risk score of 0 , the initiation of antifungal therapy within $72 \mathrm{hrs}$ after the onset of fever had no effect on patient prognosis $(5.5 \%$ vs $6.1 \%$, p= 0.877), but in patients with any risk factors, there was a statistically significant difference in patient prognosis between the patients with the initiation of antifungal therapy within $72 \mathrm{hrs}$ after the onset of fever and the patients without such initiation $(23.1 \%$ vs $30.4 \%, \mathrm{p}=0.023)$.

\section{Discussion}

The patients with hematological malignancies have a higher morbidity and mortality of bloodstream infections due to underlying immunodeficiency, cytotoxic treatment, and frequent invasive measures, and may concurrently have multiple pathogen infections. The mortality of the patients with mixed bacteria and fungi BSI have a mortality of $43 \%$ to $78 \%,{ }^{18-20}$ which is much higher than that of simple bacterial/fungal infection. In this study, we analyzed the clinical features of $1033 \mathrm{HM}$ patients with proven BSI and found that existing IFD risk scores were not accurate enough in distinguishing patients evidently benefiting from antifungal prophylaxis, so we examined the high-risk factors of IFD in case of bloodstream infections and the prognostic factors of IFD patients. In addition, we validated the impact of the time of initiating the antifungal therapy on the prognosis of HM patients with BSI bearing a high risk for IFD, providing a reference for clinical initiation of antifungal therapy in this population.

Studies have shown that the incidence of IFD in HM patients ranged from $2.1 \%$ to $7.7 \%,{ }^{5,7,17}$ which was relatively high in MDS/AML patients and the highest in patients receiving hematopoietic stem cell transplantation. Autopsies found that the incidence of IFD in HM patients was as high as $31 \%$ to $35 \%,{ }^{21-23}$ and the patients with Aspergillus infection accounted for half. ${ }^{21}$ Because of the failure to timely assess patients' lung conditions, the diagnosis in a large number of IFD patients has been missed in the clinical practice. The present study found that, in patients with HM who developed BSI, the incidence of IFD was $11.2 \%$, which was much higher than that of HM patients who had been treated with conventional chemotherapy and hematopoietic stem cell transplantation, ${ }^{7,17} \mathrm{HU}$ et al found that bacterial sepsis increased the incidence of invasive pulmonary fungal infection in HM patients who had not received hematopoietic stem cell transplantation. ${ }^{12}$ A Japanese study is the first report to identify bacteremia following febrile episodes during neutropenia as a predictive factor for IFD in pediatric patients with hematologic or malignant disease. ${ }^{24}$ The etiology of IFD after bacteremia remains unclear. Existing studies have suggested that bacteremia following febrile episodes during neutropenia appeared to be an independent predictive factor of IFI from

Table 6 Analysis of Prognostic Factors After the IFD Development in HM Patients with BSI

\begin{tabular}{|c|c|c|c|c|c|c|}
\hline \multirow[t]{2}{*}{ Prognostic Factors } & \multirow[t]{2}{*}{$N(n=\mid 16)$} & \multirow{2}{*}{$\begin{array}{l}\text { Number of Deaths(n)/ } \\
\text { Incidence }(\%)(n=47)\end{array}$} & \multicolumn{2}{|l|}{ Univariate Analysis } & \multicolumn{2}{|l|}{ Multivariate Analysis } \\
\hline & & & OR $(95 \% \mathrm{Cl})$ & P value & OR $(95 \% \mathrm{Cl})$ & $P$ value \\
\hline Age $>45$ years & $50(43.1 \%)$ & $26(55.3 \%)$ & $2.321(1.087-4.959)$ & 0.028 & $4.500(1.220-16.598)$ & 0.024 \\
\hline Relapsed or uncontrolled malignancy & $85(73.3 \%)$ & $41(87.2 \%)$ & $3.883(1.446-10.421)$ & 0.005 & $4.405(0.449-43.169)$ & 0.203 \\
\hline Renal inadequacy & $20(17.2 \%)$ & 15 (31.9\%) & $6.000(2.002-17.980)$ & 0.001 & $0.700(0.119-4.130)$ & 0.694 \\
\hline Charlson Comorbidity index $>3$ & $25(21.6 \%)$ & $16(34.0 \%)$ & $3.44 \mid(1.365-8.674)$ & 0.007 & $3.288(0.708-|5.27|)$ & 0.129 \\
\hline Pitt bacteremia score $>3$ & $41(35.3 \%)$ & $26(55.3 \%)$ & $4.457(1.981-10.030)$ & 0.000 & $3.899(1.080-14.082)$ & 0.038 \\
\hline Induction/re-induction chemotherapy & $75(64.7 \%)$ & $36(76.6 \%)$ & $2.517(1.102-5.751)$ & 0.026 & $0.601(0.090-4.015)$ & 0.599 \\
\hline Central-venous catheter & $56(48.3 \%)$ & $17(36.2 \%)$ & $0.436(0.203-0.934)$ & 0.031 & $0.342(0.094-1.237)$ & 0.102 \\
\hline Use of vasopressors & $41(35.3 \%)$ & $33(70.2 \%)$ & $17.973(6.838-47.243)$ & 0.000 & $16.35 \mid(3.774-70.846)$ & 0.000 \\
\hline $\mathrm{HB}<55 \mathrm{~g} / \mathrm{L}$ & $61(52.6 \%)$ & $30(63.8 \%)$ & $2.163(1.011-4.630)$ & 0.045 & $2.64 \mid(0.672-10.379)$ & 0.164 \\
\hline PT $\geq 16 \mathrm{sec}$ & 40 (34.5\%) & $25(53.2 \%)$ & $4.091(1.821-9.193)$ & 0.000 & $3.805(1.026-14.114)$ & 0.046 \\
\hline Antifungal therapy within $72 \mathrm{hrs}$ & $55(47.4 \%)$ & $13(27.7 \%)$ & $0.246(0.110-0.548)$ & 0.000 & $0.247(0.069-0.886)$ & 0.032 \\
\hline
\end{tabular}


Table 7 Effect of Antifungal Therapy Within 72 hrs After Giving Different Risk Scores in Patients with HM Complicated with BSI

\begin{tabular}{|l|l|l|l|l|}
\hline $\begin{array}{l}\text { Risk } \\
\text { Score }\end{array}$ & $\begin{array}{l}\text { Antifungal } \\
\text { Therapy } \\
\text { Within 72 hrs }\end{array}$ & $\begin{array}{l}\text { No. of } \\
\text { Courses (n) }\end{array}$ & $\begin{array}{l}\text { No. of } \\
\text { Deaths(n)/ } \\
\text { Incidence (\%) }\end{array}$ & P value \\
\hline 0 & $\begin{array}{l}\text { Yes } \\
\text { No }\end{array}$ & $\begin{array}{l}54 \\
229\end{array}$ & $\begin{array}{l}3(5.5 \%) \\
14(6.1 \%)\end{array}$ & 0.877 \\
\hline I-4 & $\begin{array}{l}\text { Yes } \\
\text { No }\end{array}$ & $\begin{array}{l}402 \\
348\end{array}$ & $\begin{array}{l}93(23.1 \%) \\
106(30.4 \%)\end{array}$ & 0.023 \\
\hline
\end{tabular}

those previously identified. Bacteremia occasionally progresses to sepsis, which is a systemic response associated with organ dysfunction or damage, including acute lung injury. Under such circumstances, fungi may easily invade host tissues, leading to the onset of IFD. Alternatively, changes in the levels of cytokines, chemokines, and their receptors, or the expression profile of adhesion molecules during sepsis, might influence the onset of IFD. ${ }^{24}$ Secondly, patients with bacterial infections have increased antibiotic exposure, including the initial broad-spectrum empirical treatment, which may make them susceptible to IFD. ${ }^{20}$ From the microbiological perspective, there may be synergism among different pathogens. Studies have found that in the presence of Candida albicans or its secreted cell wall polysaccharide, the drug diffusion is impaired by the mixed biofilm matrix, and the tolerance of Staphylococcus aureus to drugs becomes significantly enhanced. ${ }^{25}$ In summary, the factors mentioned above may increase the risk of IFD development in patients with HM and BSI.

IFD is an important factor in affecting the survival of HM patients. The mortality of the HM patients concomitantly with IFD was reported to be as high as $11.7-22.1 \%{ }^{8,17} \mathrm{In}$ our study, the mortality of IFD patients concomitantly with HM with BSI was $40.5 \%$, far exceeding that of the HM patients only with simple IFD infection. It means that once IFD occurred in such HM patients with concomitant IFD, the prognosis would be very poor and irreversible. Antifungal prophylaxis can reduce the morbidity and mortality of fungal infections, but excessive antifungal prophylaxis can lead to unnecessary undesirable effects and high medical costs, ${ }^{13,14}$ and increase the risk of antifungal resistance. ${ }^{15}$ The present study found that Candida tropicalis was the main strain of Candida infection in this area, and had a high resistance rate to itraconazole and fluconazole. As reported in literature, the current rate of drug resistance in fungi has increased and multidrug-resistant bacteria have emerged. ${ }^{15}$ Therefore, we must avoid the develop of IFD in such patients, and also need to accurately identify which patients have a good response to antifungal prophylaxis.

The guidelines for initiation of antifungal prophylaxis in HM patients suggest that empirical antifungal prophylaxis should be initiated in high-risk patients. ${ }^{26,27}$ Different prognostic models and risk score systems have been designed to identify the patients at high risk and who would most likely to benefit from antifungal prophylaxis. ${ }^{28-33}$ Stanzani et al reported the establishment and validation of a risk model of invasive aspergillosis in HM patients. ${ }^{28}$ However, their model only assessed the risk of mycosis and did not include the infections caused by other fungi such as Candida. Wang et al established an IFD risk score system for the HM patient on chemotherapy - the "CAESAR" score, ${ }^{17}$ and they concluded that patients at low risk could not benefit from antifungal prophylaxis. Using the "CAESAR" score system, we found that the system could distinguish between high-risk and lowrisk patients, but could not distinguish between the middle-risk and low-risk patients. In addition, the incidence of IFD in HM patients with the same risk score was higher than the incidence reported in the literature. For HM patients with BSI, even if the "CAESAR" score indicated a low risk, they might still benefit from prophylactic antifungal therapy. Therefore, "CAESAR" score cannot be applied to the IFD prophylaxis for HM patients with BSI.

We investigated the risk factors for IFD in such patients, and found that the HM patients who developed BSI had an increased probability of developing IFD if the following were satisfied: age $>45$ years old, severe granulocytopenia during the fever, hypoproteinemia and use of vasopressors. Age, granulocytopenia, and hypoproteinemia are independent risk factors for the candidemia in HM patients ${ }^{34,35}$ and can be used as predictors of IFD development. ${ }^{17}$ The proportion of developing shock was significantly increased in HM patients with mixed bacteria and fungi BSI. ${ }^{6,18}$ Septic shock was an independent risk factor for mixed Candida/bacterial BSI. ${ }^{19}$ In our study, $35.5 \%$ of patients in the IFD group required vasoactive drugs to correct the shock, and $18.9 \%$ in the non-IFD group, which demonstrated an increase in the incidence of IFD when the HM patients concomitantly developed BSI. Therefore, we designed a risk scoring system and verified that, for HM patients with BSI, the higher the score, the greater the likelihood of IFD. Those patients with high scores need to be treated as early as possible.

For HM patients with BSI and proven IFD, the mortality of the mixed bacterial/fungal BSI patients with malignant hematology was $43-78 \%,{ }^{18-20}$ much higher than that of the patients 
with simple bacterial/fungal infection, which was consistent with the mortality $(40.5 \%)$ in the BSI and HM patients with concomitant IFD in our study. The prognostic factors affecting such patients in the present study included age $>45$ years, Pitt bacteremia index score $>3$ points, use of vasoactive drugs, abnormal coagulation function ( $\mathrm{PT} \geq 16 \mathrm{~s}$ ), and initiation of antifungal therapy within $72 \mathrm{hrs}$ after the onset of fever. Among them, age, Pitt bacteremia score, use of vasoactive drugs, and abnormal coagulation function have been found by existing studies as risk factors for candidemia or IFD prognosis. $6,8,36,37$ The initiation of antifungal therapy within $72 \mathrm{hrs}$ after the onset of fever is the only protective factor and the only indicator, which may affect the prognosis of IFD development in HM patients with BSI.

Our research has found that early application of antifungal therapy within $72 \mathrm{hrs}$ after the onset of fever can significantly improve the prognosis in HM and BSI patients with proven IFD. However, it is often difficult to obtain the evidence of typical CT scan results or mycological criteria for the diagnosis of proven or probable IFD. Therefore, we investigated that if patients with HM and high risk of IFD could benefit from early initiation of antifungal therapy. The result showed that those patients with age $>45$ years, profound neutropenia, hypoproteinemia, or use of vasopressors drugs have higher survival rate if the antifungal therapy within $72 \mathrm{hrs}$ after the onset of fever. The results indicated that antifungal therapy should be initiated earlier in HM with high risk of IFD. This is a practically made decision on clinical practise. Most of bacteremia can be proven within 48 hrs by using the continuous blood culture detection system in most hospitals in China. Which means, after the onset of fever, most BSI can be proven within $72 \mathrm{hrs}$. Therefore, for patients with high-risk factors of IFD, early application of antifungal therapy can be achieved to improve the patient's prognosis.

\section{Conclusion}

As far as we know, the study may be the first report to investigate the risk prediction and prognosis of IFD in HM patients complicated with BSI. The morbidity and mortality of IFD increase significantly in HM patients with BSI than not. Several independent risk factors as well as prognosis of IFD had been identified. For HM patients with risk factors of IFD, the initiation of antifungal therapy within $72 \mathrm{hrs}$ after the onset of fever may improve the prognosis. Our research also has limitations. The retrospective study design prevented more detailed data collection to include other physiologic and social characteristics which may be pertinent to the outcomes. A prospective research would be designed to further confirm the point that early application of antifungal therapy within $72 \mathrm{hrs}$ after the onset of fever can significantly improve the prognosis in HM patients with BSI and IFD risk factors.

\section{Acknowledgments}

This work was supported by National Natural Science Foundation of China (NSFC) (Grant No. 81670203 and No.81870166) for Jing Liu; National Natural Science Foundation of China (NSFC) (Grant No. 81870165) for Xin Lin; and the Fundamental Research Funds for the Central Universities of Central South University $2015 z z t s 321$.

\section{Disclosure}

The authors report no conflicts of interest in this work.

\section{References}

1. Marin M, Gudiol C, Ardanuy C, et al. Bloodstream infections in neutropenic patients with cancer: differences between patients with haematological malignancies and solid tumours. $J$ Infect. 2014;69 (5):417-423. doi:10.1016/j.jinf.2014.05.018

2. Trecarichi EM, Pagano L, Candoni A, et al. Current epidemiology and antimicrobial resistance data for bacterial bloodstream infections in patients with hematologic malignancies: an Italian multicentre prospective survey. Clin Microbiol Infect. 2015;21(4):337-343. doi:10.1016/j.cmi.2014.11.022

3. Nørgaard M, Larsson H, Pedersen G, Schønheyder HC, Sørensen HT. Risk of bacteraemia and mortality in patients with haematological malignancies. Clin Microbiol Infect. 2006;12(3):217-223. doi:10.1111/ j.1469-0691.2005.01298.x

4. Tang Y, Cheng Q, Yang Q, et al. Prognostic factors and scoring model of hematological malignancies patients with bloodstream infections. Infection. 2018;46(4):513-521. doi:10.1007/s15010-018-1151-3

5. Pagano L, Caira M, Candoni A, et al. The epidemiology of fungal infections in patients with hematologic malignancies: the SEIFEM-2004 study. Haematologica. 2006;91(8):1068-1075.

6. Bassetti M, Righi E, Ansaldi F, et al. A multicenter study of septic shock due to candidemia: outcomes and predictors of mortality. Intens Care Med. 2014;40(6):839-845. doi:10.1007/s00134-014-3310-z

7. Sun Y, Meng F, Han M, et al. Epidemiology, management, and outcome of invasive fungal disease in patients undergoing hematopoietic stem cell transplantation in China: a multicenter prospective observational study. Biol Blood Marrow Transplant. 2015;21 (6):1117-1126. doi:10.1016/j.bbmt.2015.03.018

8. Hsu LY, Lee DG, Yeh SP, et al. Epidemiology of invasive fungal diseases among patients with haematological disorders in the Asia-Pacific: a prospective observational study. Clin Microbiol Infect. 2015;21(6):594-597. doi:10.1016/j.cmi.2015.02.019

9. Freifeld AG, Bow EJ, Sepkowitz KA, et al. Clinical practice guideline for the use of antimicrobial agents in neutropenic patients with cancer: 2010 update by the Infectious Diseases Society of America. Clin Infect Dis. 2011;52(4):e56-e93. doi:10.1093/cid/cir073

10. Mousset S, Buchheidt D, Heinz W, et al. Treatment of invasive fungal infections in cancer patients-updated recommendations of the Infectious Diseases Working Party (AGIHO) of the German Society of Hematology and Oncology (DGHO). Ann Hematol. 2014;93 (1):13-32. doi:10.1007/s00277-013-1867-1 
11. De Pauw B, Walsh TJ, Donnelly JP, et al. Revised definitions of invasive fungal disease from the European Organization for Research and Treatment of Cancer/Invasive Fungal Infections Cooperative Group and the National Institute of Allergy and Infectious Diseases Mycoses Study Group (EORTC/MSG) Consensus Group. Clin Infect Dis. 2008;46(12):1813-1821. doi:10.1086/588660

12. $\mathrm{Hu} \mathrm{R}$, Jiang $\mathrm{X}, \mathrm{Wu}$ Y. Risk factors for invasive pulmonary fungal infection in patients with hematological malignancies not receiving hematopoietic stem cell transplant. Neoplasma. 2012;59 (06):669-675. doi:10.4149/neo_2012_085

13. Cordonnier C, Pautas C, Maury S, et al. Empirical versus preemptive antifungal therapy for high-risk, febrile, neutropenic patients: a randomized, controlled trial. Clin Infect Dis. 2009;48 (8):1042-1051. doi:10.1086/599079

14. Des Champs-Bro B, Leroy-Cotteau A, Mazingue F, et al. Invasive fungal infections: epidemiology and analysis of antifungal prescriptions in onco-haematology. J Clin Pharm Ther. 2011;36(2):152-160. doi:10.1111/jcp.2011.36.issue-2

15. Verweij PE, Mellado E, Melchers WJ. Multiple-triazole-resistant aspergillosis. N Engl J Med. 2007;356(14):1481-1483. doi:10.1056/ NEJMc061720

16. Baden LR, Bensinger W, Angarone M, et al. Prevention and treatment of cancer-related infections. J Natl Compr Canc Netw. 2012;10 (11):1412-1445. doi:10.6004/jnccn.2012.0146

17. Wang $\mathrm{L}$, Wang $\mathrm{Y}, \mathrm{Hu} \mathrm{J}$, et al. Clinical risk score for invasive fungal diseases in patients with hematological malignancies undergoing chemotherapy: China assessment of antifungal therapy in hematological diseases (CAESAR) study. Front Med. 2019;13(3):365-377. doi:10.1007/s11684-018-0641-0

18. Bouza E, Burillo A, Muñoz P, Guinea J, Marín M, Rodríguez-Créixems M. Mixed bloodstream infections involving bacteria and Candida spp. J Antimicrob Chemother. 2013;68(8):1881-1888. doi:10.1093/jac/dkt099

19. Kim SH, Yoon YK, Kim MJ, Sohn JW. Risk factors for and clinical implications of mixed Candida/bacterial bloodstream infections. Clin Microbiol Infect. 2013;19(1):62-68. doi:10.1111/j.1469-0691.2012.03906.x

20. Reno J, Doshi S, Tunali AK, et al. Epidemiology of methicillinresistant staphylococcus aureus bloodstream coinfection among adults with candidemia in Atlanta, GA, 2008-2012. Infect Control Hosp Epidemiol. 2015;36(11):1298-1304. doi:10.1017/ice.2015.185

21. Kami M, Machida U, Okuzumi K, et al. Effect of fluconazole prophylaxis on fungal blood cultures: an autopsy-based study involving 720 patients with haematological malignancy. $\mathrm{Br} J$ Haematol. 2002;117(1):40-46. doi:10.1046/j.1365-2141.2002.03414.x

22. Lewis RE, Cahyame-Zuniga L, Leventakos K, et al. Epidemiology and sites of involvement of invasive fungal infections in patients with haematological malignancies: a 20-year autopsy study. Mycoses. 2013;56(6):638-645. doi:10.1111/myc.2013.56.issue-6

23. Chamilos G, Luna M, Lewis RE, et al. Invasive fungal infections in patients with hematologic malignancies in a tertiary care cancer center: an autopsy study over a 15-year period (1989-2003). Haematologica. 2006;91(7):986-989.

24. Sano H, Kobayashi R, Suzuki D, Kishimoto K, Yasuda K, Kobayashi K. Bacteremia during neutropenia is a predictive factor for invasive fungal infection in children. Pediatr Int. 2013;55 (2):145-150. doi:10.1111/ped.2013.55.issue-2
25. Kong EF, Tsui C, Kucharikova S, Andes D, Van Dijck P, Jabra-Rizk MA. Commensal protection of staphylococcus aureus against antimicrobials by Candida albicans Biofilm Matrix. mBio. 2016;7(5). doi:10.1128/mBio.01365-16

26. Colombo AL, de Almeida JJ, Slavin MA, Chen SC, Sorrell TC. Candida and invasive mould diseases in non-neutropenic critically ill patients and patients with haematological cancer. Lancet Infect Dis. 2017;17(11):e344-56. doi:10.1016/S1473-3099(17)30304-3

27. Pagano L, Busca A, Candoni A, et al. Risk stratification for invasive fungal infections in patients with hematological malignancies: SEIFEM recommendations. Blood Rev. 2017;31(2):17-29. doi:10.1016/j. blre.2016.09.002

28. Stanzani M, Lewis RE, Fiacchini M, et al. A risk prediction score for invasive mold disease in patients with hematological malignancies. PLoS One. 2013;8(9):e75531. doi:10.1371/journal.pone.0075531

29. Rodríguez Veiga R, Montesinos P, García E, et al. Validation of a multivariable prediction model for post-engraftment invasive fungal disease in 465 adult allogeneic hematopoietic stem cell transplant recipients. Mycoses. 2019;62(5):418-427. doi:10.1111/myc.2019.62.issue-5

30. Takaoka K, Nannya Y, Shinohara A, Arai S, Nakamura F, Kurokawa M. A novel scoring system to predict the incidence of invasive fungal disease in salvage chemotherapies for malignant lymphoma. Ann Hematol. 2014;93(10):1637-1644. doi:10.1007/ s00277-014-2093-1

31. Montesinos P, Rodriguez-Veiga R, Boluda B, et al. Incidence and risk factors of post-engraftment invasive fungal disease in adult allogeneic hematopoietic stem cell transplant recipients receiving oral azoles prophylaxis. Bone Marrow Transplant. 2015;50(11):1465-1472. doi:10.1038/bmt.2015.181

32. Parody R, Martino R, Sánchez F, Subirá M, Hidalgo A, Sierra J. Predicting survival in adults with invasive aspergillosis during therapy for hematological malignancies or after hematopoietic stem cell transplantation: single-center analysis and validation of the Seattle, French, and Strasbourg prognostic indexes. Am J Hematol. 2009;84 (9):571-578. doi:10.1002/ajh.v84:9

33. Muskett H, Shahin J, Eyres G, Harvey S, Rowan K, Harrison D. Risk factors for invasive fungal disease in critically ill adult patients: a systematic review. Crit Care. 2011;15(6):R287. doi:10.1186/cc10574

34. Gamaletsou MN, Walsh TJ, Zaoutis T, et al. A prospective, cohort, multicentre study of candidaemia in hospitalized adult patients with haematological malignancies. Clin Microbiol Infect. 2014;20(1): O50-7. doi:10.1111/1469-0691.12312

35. Gao L, Sun Y, Meng F, et al. Antifungal prophylaxis of patients undergoing allogenetic hematopoietic stem cell transplantation in China: a multicenter prospective observational study. J Hematol Oncol. 2016;9(1):97. doi:10.1186/s13045-016-0305-y

36. Criscuolo M, Marchesi F, Candoni A, et al. Fungaemia in haematological malignancies: SEIFEM-2015 survey. Eur J Clin Invest. 2019;49(5):e13083. doi:10.1111/eci.2019.49.issue-5

37. Vaquero-Herrero MP, Ragozzino S, Castaño-Romero F, et al. The Pitt bacteremia score, Charlson comorbidity index and chronic disease score are useful tools for the prediction of mortality in patients with Candida bloodstream infection. Mycoses. 2017;60(10):676-685. doi:10.1111/myc.2017.60.issue-10

\section{Publish your work in this journal}

Cancer Management and Research is an international, peer-reviewed open access journal focusing on cancer research and the optimal use of preventative and integrated treatment interventions to achieve improved outcomes, enhanced survival and quality of life for the cancer patient.

The manuscript management system is completely online and includes a very quick and fair peer-review system, which is all easy to use. Visit http://www.dovepress.com/testimonials.php to read real quotes from published authors. 